\title{
Case Report: Misdiagnosis of Post-traumatic Osteomyelitis: A Case Report
}

\author{
Salman Ghaffari ${ }^{1}$ (D), Mehran Razavipour ${ }^{1}$ (D), Iman Sadeghian ${ }^{2 *}$ (D), Shahin Talebi ${ }^{1}$ (D) \\ 1. Orthopedic Research Center, Mazandaran University of Medical Science, Sari, Iran \\ 2. Orthopedic Surgeon, Semnan University of Medical Science, Semnan, Iran.
}

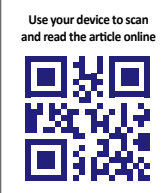

Citation Ghaffari S, Razavipour M, Sadeghian I, Talebi S. Misdiagnosis of Post-traumatic Osteomyelitis: A Case Report. Journal of Research in Orthopedic Science. 2021; 8(2):107-110. http://dx.doi.org/10.32598/JROSJ.8.2.751.1

http://dx.doi.org/10.32598/JROSJ.8.2.751.1

\section{(c) $(1) \Theta$}

Article info:

Received: 04 Jan 2021

Revised: 26 Feb 2021

Accepted: 06 Mar 2021

Available Online: 01 May 2021

Keywords:

Chronic osteomyelitis, Hodgkin lymphoma, Left tibia fibula, Tibia fracture

\begin{abstract}
A B S T RA C T
Chronic osteomyelitis is a severe and persistent condition that engages bone and bone marrow. The infection can be limited to the bone, or it can propagate to the bone marrow, the periosteum, and the surrounding soft tissues. It represents a major financial every health system and impacts the quality of life of the affected patients. Diagnosing chronic osteomyelitis remains difficult. Accordingly, laboratory and imaging studies are necessary in this respect. A 49-year-old man after high energy trauma underwent debridmant and fixation. After 3 years' patient admitted in infection ward with fever presentation. The clinical examination didn't reveal any sign of localization of fever. Chronic osteomyelitis is not always associated with fever in this situation Other diagnoses should be considered after role out other disease osteomyelitis
\end{abstract}

\section{Introduction}

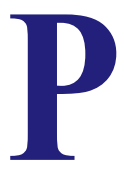

ost-Traumatic Osteomyelitis (PTO), also called fracture-related, is an osteomyelitis deep infection. The incidence rate of post-fracture PTO is reported to be $1 \%-19 \%$ [1]. The distribution of PTO depends on the contaminated soft tissue, open fracture, and high energy trauma. This infection is among the worst complications of orthopedic surgery in chronic osteomyelitis. It is associated with a long-term infection of bone marrow; osteomyelitis is characterized by lowgrade inflammation induced by persistent infection with sequestrum or fistulous tract [2]. The clinical symptoms of osteomyelitis include chronic pain, persistent sinus tract or wound drainage, malaise, and sometimes fever [3]. The diagnosis of chronic osteomyelitis remains difficult and laboratory and imaging studies are required in this respect.

\section{Case Presentation}

In 2015, a 49-year-old man, after high energy trauma, encountered a left tibia fibula fracture with excessive soft tissue damage. The patient was transferred to the emergency department. The patient also experienced a right

\section{* Corresponding Author:}

Iman Sadeghian, MD.

Address: Orthopedic surgeon, Semnan University of Medical Science, Semnan, Iran

Phone: +98 (11) 33377169

E-mail: iman.sadeghian@gmail.com 


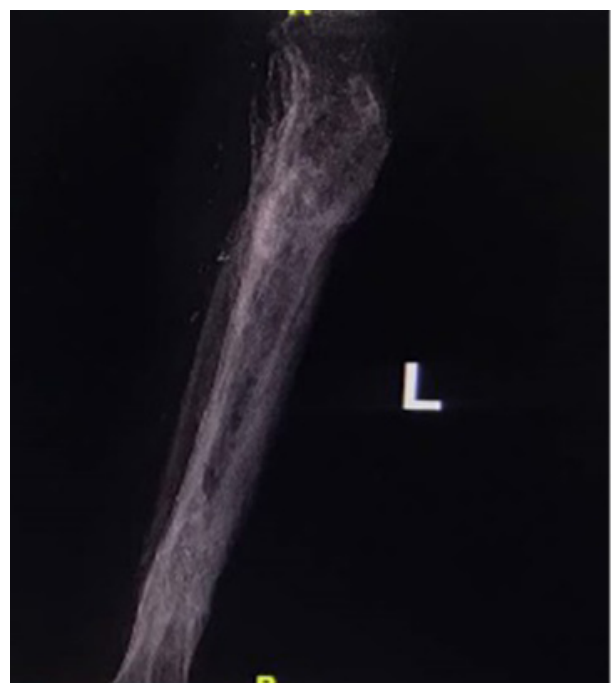

Figure 1. Left tibia X-ray of the patient

femoral fracture that treatment with the plate. On the first day of admission, an external fixator was applied to the study patient. After 3 days of hospitalization, the patient underwent surgery due to necrotizing fasciitis. Furthermore, the anterior and lateral compartments were debridement. The reported patient was discharged with a non-functional leg.

Three years later, the patient was admitted to the infection ward with a fever presentation. The clinical examination revealed no sign of the localization of fever. The left leg skin was clear and without evidence of fistula. The following laboratory studies were performed: Complete Blood Count (CBC); Peripheral Blood Smear

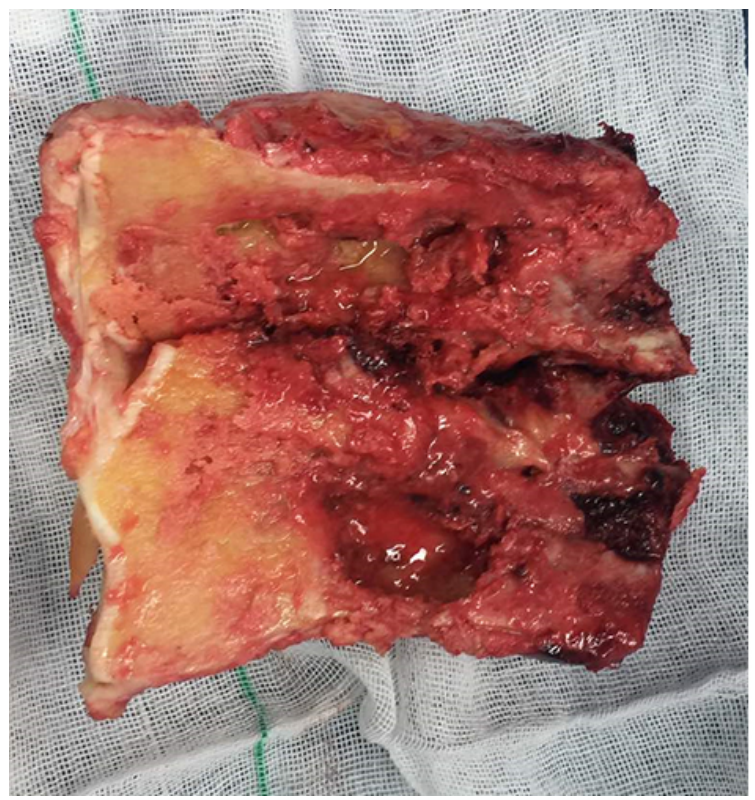

Figure 2. Intraoperative picture of the patient's bone fistula

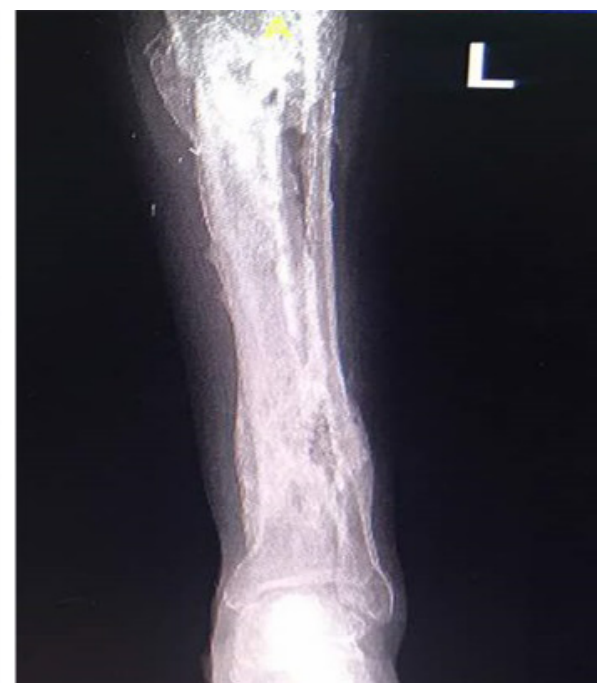

Orthopedic Science

(PBS); inflammatory indexes, e.g. Erythrocyte Sedimentation Rate (ESR), C-Reactive Protein (CRP), Serum Protein Electrophoresis (SPEP); serum chemistry; urine and blood culture; throat and urethral swab; serology for viral hepatitis, Human Immunodeficiency Virus (HIV), and brucellosis infection (Table 1). After conducting these investigations, the patient dismissed himself to the hospital. The patient was re-admitted to the orthopedic ward to rule out osteomyelitis. In addition to previous laboratory tests, chest X-ray, left tibia X-ray (Figure 1), skull radiographic, abdominal ultrasonography, and a total body Computed Tomography (CT) scan was performed in the patient. CT scan findings have led the orthopedic surgeon to chronic osteomyelitis. Hematologist

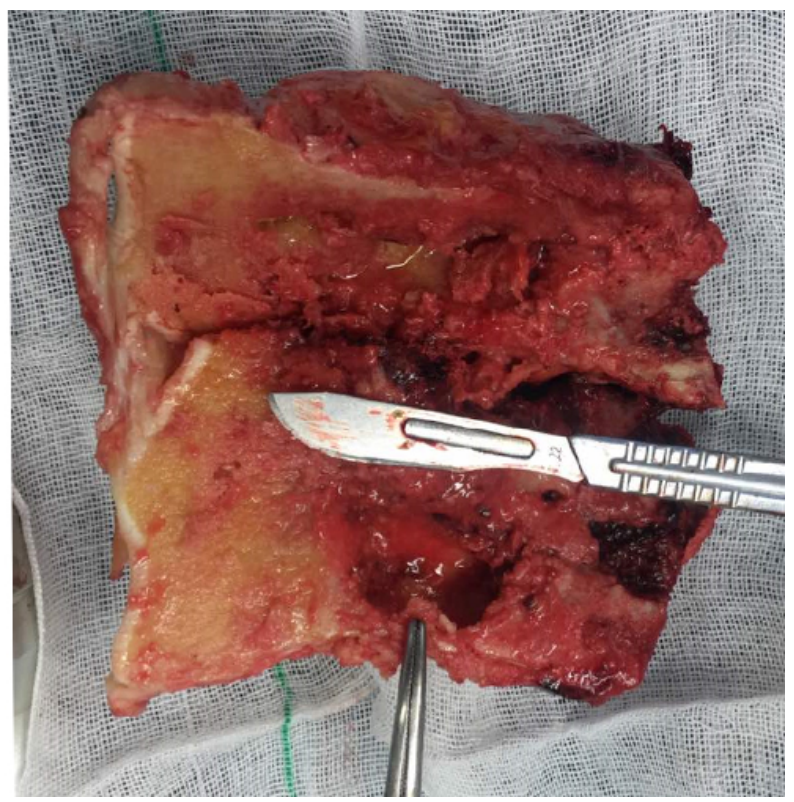

Orthopedic Science 
Table 1. Laboratory test findings at the patient's first admission

\begin{tabular}{cc}
\hline Tests & Values \\
\hline White blood cells $\left(\times 10^{3} / \mu \mathrm{L}\right)$ & 8.81 \\
\hline Red blood cells $\left(\times 10^{6} / \mu \mathrm{L}\right)$ & 3.84 \\
\hline Platelets $\left(\times 10^{3} / \mu \mathrm{L}\right)$ & 112 \\
\hline Hemoglobin $(\mathrm{g} / \mathrm{dL})$ & 10.0 \\
\hline Hematocrit $(\%)$ & 38.3 \\
\hline Neutrophils $(\%)$ & 71.9 \\
\hline Lymphocytes $(\%)$ & 19.7 \\
\hline Monocytes $(\%)$ & 7.5 \\
\hline Eosinophils $(\%)$ & 0.6 \\
\hline Basophils $(\%)$ & 0.3 \\
\hline ESR $(\mathrm{mm} / \mathrm{h})$ & 55 \\
\hline CRP $(\mathrm{mg} / \mathrm{L})$ & 60 \\
\hline
\end{tabular}

and infectious disease specialist consultations were also conducted. Both experts suggested no evidence of infectious disease or malignancy. The patient was transferred to the operating room for below-knee amputation. Numerous bone tracts were detected in the patient (Figure 2). The patient's body temperature was under 38 for 3 days; then, a fever of $>40^{\circ}$ was generated in him again.

The explored patient dismissed himself again. After two months, the reported patient was re-admitted with high fever and inguinal lymphadenopathy. A biopsy of the inguinal lymph node was performed. The pathologist reported classical Hodgkin lymphoma mixes cellularity. The patient expired 10 days after Intensive Care Unit (ICU) admission

\section{Discussion}

The diagnosis of chronic osteomyelitis remains a challenge. In our case, the first diagnosis based on the patient's history and imaging findings was chronic osteomyelitis. Chronic osteomyelitis is characterized by alternating periods of pain, swelling, and sinus tract drainage. The reported patient presented a high fever; however, CT scan and post-surgery findings suggested osteomyelitis.

Fever of Unknown Origin (FUO) was defined by Petersdorf and Beeson as a temperature above $38.30 \mathrm{C}$ on several occasions over $>3$ weeks; for which, no diagnosis must have been reached despite 1 week of inpatient investigation $[4,5]$. They observed that FUOs were caused by an infection in $36 \%$ of cases, malignancy in $19 \%$, and collagen vascular disease in $19 \%$, and miscellaneous causes in $19 \%$. No cause was detected in $7 \%$ of the explored cases [6].

Hodgkin lymphoma is a common lymphoid malignancy. This condition originates from white blood cells called lymphocytes [7]. It usually initiates in an area within the lymphatic system spread of disease from one lymph node group to another. B symptoms; fevers, chills, night sweats, or unexplained weight loss of $>10 \%$ of body weight are frequent in this case; however, only fever was present in our patient. The patient presents spiky over 40 degrees in patients with advanced-stage. It accounts for approximately $0.5 \%$ of newly diagnosed cases of cancer in the United States and about $0.2 \%$ of all cancer-induced deaths [8].

\section{Conclusion}

In our experience, at the first time, the patient's fever should have not been linked to the amputation and chronic osteomyelitis. Chronic osteomyelitis is not always associated with fever in such situations. Other diagnoses should be considered after ruling out other conditions, including osteomyelitis. 


\section{Ethical Considerations}

\section{Compliance with ethical guidelines}

There were no ethical considerations to be considered in this research.

\section{Funding}

This research did not receive any grant from funding agencies in the public, commercial, or non-profit sectors.

\section{Authors' contributions}

All authors equally contributed to the designing of the scientific proposal, data collection, and manuscript drafting. The final manuscript was reviewed and approved by all authors.

\section{Conflict of interest}

There authors declared no conflicts of interest.

\section{References}

[1] Barakat A, Schilling WHS, Sharma S, Guryel E, Freeman R. Chronic osteomyelitis: A review on current concepts and trends in treatment. Orthop Trauma. 2019; 33(3):181-7. [DOI:10.1016/j.mporth.2019.03.005]

[2] Govaert GA, IJpma FF, McNally M, McNally E, Reininga IH, Glaudemans AW. Accuracy of diagnostic imaging modalities for peripheral post-traumatic osteomyelitis-a systematic review of the recent literature. Eur J Nucl Med Mol Imaging. 2017; 44(8):1393-407. [DOI:10.1007/s00259-017-3683-7]

[3] Kudva A, Kamath AT, Dhara V, Ravindranath V. Chronic recurrent osteomyelitis: A surgeon's enigma. J Oral Pathol Med. 2019; 48(2):180-4. [DOI:10.1111/jop.12814]

[4] Emecen AN, Burcu I, Aslan A, Arslan F, Vahaboğlu H. Misleading radiological appearances for fever of unknown origin: Beyond terminal ileitis. Med J Infect Microbes Antimicrob. 2018; 7:5. [DOI:10.4274/mjima.2018.5]

[5] Horowitz HW. Fever of unknown origin or fever of too many origins? N Engl J Med. 2013; 368(3):197-9. [DOI:10.1056/NEJMp1212725]

[6] Roth AR, Basello GM. Approach to the adult patient with fever of unknown origin. Am Fam Physician. 2003; 68(11):2223-8. https://www.aafp.org/afp/2003/1201/ afp20031201p2223.pdf

[7] Shanbhag S, Ambinder RF. Hodgkin lymphoma: A review and update on recent progress. CA Cancer J Clin. 2018; 68(2):116-32. [DOI:10.3322/caac.21438]

[8] Sud A, Hemminki K, Houlston RS. Candidate gene association studies and risk of Hodgkin lymphoma: A systematic review and meta-analysis. Hematol Oncol. 2017; 35(1):34-50 [DOI:10.1002/hon.2235] [PMID] [PMCID] 